\section{An unexpected fever post serogroup B meningococcal sepsis}

\author{
Daniele Donà, 1 Elisa Gnoato,2 \\ Carlo Giaquinto, ${ }^{1}$ Carlo Moretti ${ }^{3}$ \\ 1Pediatric Infectious Diseases Division, \\ 2Pediatric Pulmonology and Allergy \\ Division, 3Pediatric Diabetology Division, \\ Department for Woman and Child \\ Health, University of Padua, Italy
}

\begin{abstract}
This case report describes an invasive meningococcal group B infection followed by the development of Kawasaki disease (KD) complicated by macrophage activation syndrome (MAS) in a 2-year-old child. The presented case indicates the possible etiologic relationship between meningococcal sepsis and KD as support of bacterial toxin induced theory. It's important to maintain a high grade of suspicious for KD in every relapse of fever also during convalescence phase of severe infection. Usually, initial treatment with intravenous immunoglobulin is sufficient to control the disease; but, in case of refractory KD complicated by MAS, corticosteroid therapy represents a good option inducing prompt fever resolution and clinical improvement.
\end{abstract}

\section{Introduction}

Kawasaki disease (KD) is an acute febrile necrotizing vasculitis of medium and small-size arteries, which usually occurs in children under 5 years of age.1,2 At present, an unusual immune activation following a bacterial toxins is considered to be the basis of KD's pathology. Staphylococci, streptococci are some of the infectious agents most implicated with the disease, while an association with meningococcal disease is poorly documented.1,3-7 This is the case of a 2-year-old boy with Neisseria meningitidis serogroup B meningococcemia who also developed KD complicated by macrophage activation syndrome (MAS) efficaciously treated with intravenous methylprednisolone (IVMP) after intravenous immunoglobulin (IVIG) treatment failure. Our aim with this report is to increase pediatricians' awareness about the importance of a high grade of suspect for KD in rare and unexpected situations, since more and more evidence suggests that delaying the diagnosis, and consequentially therapy, lead to development of coronary artery aneurysms, increase treatment failure rate and complications. ${ }^{8-12}$

\section{Case Report}

A previously healthy 2-year-old Caucasian boy was brought to the Emergency Room (ER) of a local hospital because of few hour history of high fever, not relieved by antipyretic treatment. Physical examination revealed a febrile [body temperature (BT) of $39.2^{\circ} \mathrm{C}$ ], severely ill, extremely irritable child with heart rate of $170 / \mathrm{min}$, blood pressure of $98 / 51 \mathrm{mmHg}$ and petechial lesions disseminated on the torso and limbs. Suspecting meningococcemia, intravenous (IV) fluids and ceftriaxone had been immediately administered. Due to hypotension, despite massive IV fluids resuscitation, after neurological deterioration the child required inotropic support, intubation and mechanical ventilation before being transferred to the pediatric Intensive Care Unit (PICU) of our Pediatrics Department. Neisseria meningitidis serogroup B was isolated from the blood culture drawn in the ER while a cerebrospinal fluid sample was normal on biochemistry, microscopy and culture.

After PICU admission antibiotic therapy with ceftriaxone was continued with gradual improvement of the patient's general conditions and inflammatory markers levels [Creactive protein (CRP) decreased from 345 $\mathrm{mg} / \mathrm{L}$ after admission to $48.4 \mathrm{mg} / \mathrm{L}$ on day 9 (normal value up to $6 \mathrm{mg} / \mathrm{L}$ )]. On day 5 inotropic agents were stopped and on day 7 the child was extubated. Despite the general improvement, he continued to present spikes of fever (BT of $38.5^{\circ} \mathrm{C}$ ). Suspecting a hospital acquired infection, a broader antibiotic therapy with ceftriaxone, gentamicin and teicoplanin was started. Blood and urine cultures and a lumbar puncture yielded negative results. Chest X-ray and brain magnetic resonance imaging (MRI) didn't show any sign of an ongoing infection. On his transfer to the General Pediatrics Unit (GPU) on day 13, he was stable but still febrile (BT of $38.5^{\circ} \mathrm{C}$ ). Physical examination was unremarkable except for the presence of numerous cutaneous ulcers in different stages of scarring. Blood tests showed: hemoglobin (HB) $8.1 \mathrm{~g} / \mathrm{L}$, red blood cells $3,050,000 / \mathrm{mm}^{3}$, mean corpuscular volume (MCV) $80.6 \mathrm{fl}$, white blood cell (WBC) count $25,830 / \mathrm{mm}^{3}$ [polymorphonuclear

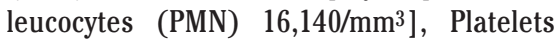
(PLT) count $1.090 .000 / \mathrm{mm}^{3}$ and stable CRP $(44.80 \mathrm{mg} / \mathrm{L})$. During the first week (days 13 20 ) in the GPU child remained febrile with BTs always $<39^{\circ} \mathrm{C}$ with one or two spikes per day. On day 21 his conditions worsened despite broad-spectrum antibiotic treatment. He became extremely irritable with more frequent (every 6-8 hours) fever spikes that reached higher temperatures $\left(\mathrm{BT}>39^{\circ} \mathrm{C}\right)$. CRP $(89.70$ $\mathrm{mg} / \mathrm{L}$ ), erythrocyte sedimentation rate (ESR) (36 $\mathrm{mm} / \mathrm{h}$ with normal value $0-15)$, Ferritin
Correspondence: Daniele Donà, Division of Pediatric Infectious Diseases, Department for Woman and Child Health, University of Padua, Via Giustiniani 3, 35124 Padua, Italy.

Tel.: +39.338.8946412

E-mail: daniele.dona@phd.unipd.it.

Key words: Neisseria meningitidis serogroup B; Kawasaki Diseases; Corticosteroid therapy macrophage activation syndrome.

Contributions: DD drafted the initial manuscript; EG, $\mathrm{CG}$ and $\mathrm{CM}$ reviewed and revised the manuscript and approved the final manuscript as submitted.

Conflict of interest: the authors declare no potential conflict of interest.

Received for publication: 20 May 2016.

Revision received: 20 0ctober 2016.

Accepted for publication: 24 October 2016.

This work is licensed under a Creative Commons Attribution NonCommercial 4.0 License (CC BYNC 4.0).

CC Copyright D. Donà et al., 2016

Licensee PAGEPress, Italy

Pediatric Reports 2016; 8:6613

doi:10.4081/pr.2016.6613

(173 ug/L with normal value 7-140), neopterine $(31.0 \mathrm{nmol} / \mathrm{L}$ with normal up to $10 \mathrm{nmol} / \mathrm{L})$ and TNF (29.8 ng/L with normal value up to 8.1 $\mathrm{ng} / \mathrm{L}$ ) confirmed the persistence of the inflammatory state. Due to the worsening of general conditions antibiotic therapy was broadened with meropenem and teicoplanin and a new set of cultures was collected. All the cultures collected resulted negative and gradually, from day 22, the child developed erythematouspapular rash, hepatomegaly, and remarkable bilateral cervical lymphadenopathy. Suspecting a viral infection, serological tests for Adenovirus, EBV, CMV and Parvovirus B19 were performed and came back negative on day 25 . Neck ultrasound showed only the presence of reactive lymph nodes. Considering the high suspicion of a vasculitis as atypical Kawasaki, an electrocardiogram and echocardiography were performed. The first was normal and the second showed: No atrial shunt, no mitral or tricuspid regurgitation, good biventricular contractility. Hyper echogenicity of mitral papillary muscles, outflows free, normal coronaries. On day 26 one dose IVIG was administered at a dose of $2 \mathrm{~g} / \mathrm{kg}$ with no effect on the fever spikes. So on day 28 IVIG administration had been repeated still with poor clinical improvement. On day 29 his blood tests showed: HB $7.4 \mathrm{~g} / \mathrm{dL}, \mathrm{RBC} 3,050,000 / \mathrm{mm}^{3}$, MCV 76.4 fl, WBC count $23,470 / \mathrm{mm}^{3}$, PMN

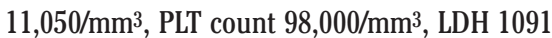


$\mathrm{U} / \mathrm{L}$, normal renal function, albumin $31 \mathrm{~g} / \mathrm{L}$, AST 86 IU/L, ALT 117 IU/L, GGT 300 U/L, sodium $135 \mathrm{mmol} / \mathrm{L}$, triglycerides $361 \mathrm{mg} / \mathrm{dL}$ (normal value $<150 \mathrm{mg} / \mathrm{dL}$ ) with coagulation pattern compatible with severe discoagulopathy with prolongation of prothrombin (15\%) and activated partial thromboplastin time (45 sec), fibrinogen $1 \mathrm{~g} / \mathrm{L}$ (normal value 1.5-4.5 $\mathrm{g} / \mathrm{L}$ ). Fever remained high $\left(\mathrm{BT}>39^{\circ} \mathrm{C}\right)$ and $\mathrm{CRP}$ elevated $(64.70 \mathrm{mg} / \mathrm{L})$. Vitamin $\mathrm{K}$ and fresh frozen plasma were administered with normalization of coagulation pattern. Bone marrow aspirate showed no signs of hemophagocytic lymphohistiocytosis. Furthermore, from day 30 the child gradually presented conjunctival injection, fissured dry lips and facial and feet edema, matching all criteria for KD. Suspecting KD unresponsive to IVIG complicated by MAS on day 32 IVMP at a dose of 30 $\mathrm{mg} / \mathrm{kg}$ for 2 consecutive days was administered.

In the following days inflammatory markers gradually decreased (CRP $3.36 \mathrm{mg} / \mathrm{L}$ on day 35 ) and fever spikes reduced. On day 37 the child finally remained afebrile. Before discharge no cardiac worsening on follow-up echocardiogram was documented.

Follow up at 3 months after the onset of his illness showed a healthy child with normal physical examination, laboratory tests, inflammatory markers and ECG were normal and follow-up echocardiogram confirmed no cardiac worsening.

\section{Discussion}

$\mathrm{KD}$ is an acute vasculitis described for the first time in Japan in 19672. Its etiology is still unknown but epidemiologic and immunologic evidence indicates that the most likely trigger agent is infectious.1,3-7 However, genetic predisposition and immune-mediated reactions have been suggested as possible etiologic factors. ${ }^{13}$ Our patient presented a serogroup B meningococcal sepsis with a history of fever, irritability, petechial rash with elevation of WBC and inflammatory markers. This infection was efficiently treated with IV antibiotic therapy, with general conditions improvement and decrease of inflammatory markers, but the child continued spiking fever correlated with KD. As suggested by other authors, $3,5,6$ the close temporal occurrence shows how the meningococcal toxin, acting as superantigen, could trigger an unusual and uncontrolled immune activation with a consequent development of an acute inflammatory vasculitis, with persistence of fever. ${ }^{3-7}$

The main challenges posed by KD are recognizing the disease in its early stages and preventing complications. ${ }^{11,13,14}$

$\mathrm{KD}$ is usually characterized by prolonged fever ( $\geq 5$ days) and at least 4 out of 5 key diagnostic features (bilateral non-exudative bulbar conjunctival injection, polymorphous exanthema, erythema and edema on the hands and feet, strawberry tongue and lip fissures, cervical lymphadenopathy $\geq 1.5 \mathrm{~cm}$ ) or fever associated with less than 4 of the diagnostic criteria and echocardiographic abnormalities of the coronary arteries. ${ }^{2}$ Fever is universal and usually began abruptly. Affected children are more irritable than expected with the degree of fever. ${ }^{2}$ In this case we thought about KD on day 22 when the pattern of fever changed and it was associated with other symptoms. However, it's likely that the KD inflammatory state had started around ten days before. Excluding fever, all other signs and symptoms required for the diagnosis can appear in different stages but, up to $20 \%$ of all KD cases present incomplete disease defined as fever without other requested clinical criteria. ${ }^{2}$ When atypical KD is suspected, in addition to prolonged fever and increased inflammatory markers $(C R P \geq 3.0 \mathrm{mg} / \mathrm{dL}$ or $\mathrm{ESR} \geq 40 \mathrm{~mm} / \mathrm{h}$ ), only three more laboratory criteria (serum albumin $\leq 3.0$ $\mathrm{g} / \mathrm{dL}$, anemia for age, increased ALT levels, PLT $\geq 450,000 / \mathrm{mm}^{3}$ after the 7 th day of fever, WBC count $\geq 15,000 / \mathrm{mm}^{3}$, and urine $\mathrm{WBC} \geq 10 /$ high power field) are sufficient to start treatment even without echocardiography. ${ }^{15}$ These criteria had already come out before day 22. Therefore, further changing in fever pattern and skin rash that were considered due to viral infections or incomplete KD, were related with MAS. For this reason high index of suspicion is imperative for any young child with prolonged fever and no clear diagnosis, especially for inpatient on convalesce phase after a severe infection. Indeed, in hospitalized children in addition to the common viral infections or illnesses caused by bacterial toxins must be considered other types of illness such as: the relapse of previous infection, drugs reaction or hospital-acquired infection. In those cases KD diagnosis could be even more challenging and time consuming.

Laboratory tests, though nonspecific, can provide diagnostic support for KD in patients with suggestive clinical features. As we found, high WBC count is typical during the acute stage with PMN predominance. Anemia with normal RBC had been found after a prolonged duration of active inflammation (HB $7.4 \mathrm{~g} / \mathrm{L}$ on day 29). Thrombocytosis, defined as PLT count 500,000 to $>1 \mathrm{million} / \mathrm{mm}^{3}$, is a characteristic feature of the later phases, usually appears in the second week, peaks in the third week and returns normal by 4 to 8 weeks after onset in uncomplicated cases. The PLT count pattern we found was characterized by thrombocytosis on day 13 followed by gradual reduction despite the ongoing inflammatory state. The most likely interpretation is that day 13 thrombocytosis was not reactive to previous infec- tious process but attributable to KD. The following thrombocytopenia was a sign of MAS, as a complication of KD treatment delay. ${ }^{16,17}$ Indeed, our patient had prolonged fever, rash, hepatomegaly and laboratory findings as thrombocytopenia, hyperferritinaemia, hypertriglyceridemia and hypofibrinogenemia which fit the MAS diagnostic criteria. The only missing finding was hemophagocytosis in bone marrow aspirate.

Time represents the key factor for successful KD treatment because delaying therapy is associated with development of coronary artery aneurysms, higher treatment failure rate and increase risk of complication, as MAS. ${ }^{8-11,17}$

In fact, $\mathrm{KD}$ represents the commonest cause of acquired heart disease in children in industrialized countries; 18 with a good prognosis if treated early. In $25-30 \%$ of untreated children coronary artery aneurysms could be present, leading to ischemic heart disease, myocardial infarction. 19-21 Regarding cardiac involvement, no coronary artery aneurysms had been reported despite the long course of symptoms and hyper echogenicity of mitral papillary muscles was the only cardiac anomaly found. These alterations are often observed during the acute phase of KD regardless of coronary involvement and,19 as in our case, usually disappear with the resolution of acute illness. ${ }^{21}$

IVIG at $2 \mathrm{~g} / \mathrm{kg}$ represents the resolving treatment for around $80-90 \%$ of patients with a clinical and biochemical remission. ${ }^{2}$ The remaining percentage, which does not become afebrile after 36 hours after completion of IVIG single infusion, could benefit from second IVIG administration. Nevertheless, only two-thirds of these patients respond to the second IVIG. ${ }^{14}$ For refractory forms, second line treatment is represented by corticosteroids, which may inhibit immune cells and inflammatory cytokines that cannot be completely suppressed by IVIG. Indeed, according to some authors, the use of intravenous methylprednisolone (IVMP) seems to be associated with a decrease in fever but its role on the progression of coronary artery anomalies is still uncertain. $22-25$

The association between MAS with rheumatic diseases, including KD has been well described by other authors.10,17,26 MAS treatment is not standardized and different agents has been suggested: IVMP, anti-TNF- and cyclosporine A.17,27

Back to our case, aside from the prolonged fever, many of the diagnostic criteria required to make KD diagnosis had appeared only long time after the fever began. This led to a delayed diagnosis and treatment with IVIG that should have been administered within the first 10 days of illness. ${ }^{2}$ Considering clinical scenario of a MAS complicating KD, IVMP were administered with dramatic clinical improve- 
ment.

\section{Conclusions}

The presented report indicates the possible etiologic relationship between invasive meningococcal serogroup B infection and KD as support of superantigen-induced theory. Every pediatrician must maintain a high grade of suspicion for KD in every case of prolonged fever and elevated inflammatory markers unresponsive to antibiotics. It's important to suspect precociously $\mathrm{KD}$, because delay in diagnosis increases the risk of IVIG treatment failure, coronary aneurysms and immunemediated complications as MAS. In this case, IVMP therapy represented a good option for treating KD complicated by MAS, inducing prompt fever resolution and clinical improvement.

\section{References}

1. Kim KY, Kim DS. Recent advances in Kawasaki disease. Yonsei Med J 2016;57:15-21.

2. Newburger JW, Takahashi M, Committee on Rheumatic Fever, Endocarditis, and Kawasaki Disease, et al. Diagnosis, treatment, and long-term management of Kawasaki disease: a statement for health professionals from the Committee on Rheumatic Fever, Endocarditis, and Kawasaki Disease, Council on Cardiovascular Disease in the Young, American Heart Association. Pediatrics 2004;114:1708-33.

3. Abe J, Kotzin BL, Jujo K. Selective expansion of $\mathrm{T}$ cells expressing T-cell receptor variable regions $\mathrm{V}$ beta 2 and $\mathrm{V}$ beta 8 in Kawasaki disease. Proc Natl Acad Sci USA 1992;89:4066-70.

4. Fretzayas A, Stabouli S, Alexaki A, Moustaki M. Meningococcal group A sepsis associated with rare manifestations and complicated by Kawasaki-like disease. Pediatr Emerg Care 2009;25:190-2.
5. Ramanan AV, Baildam EM. Kawasaki disease following meningococcal septicaemia. Arch Dis Child 2002;87:170-1.

6. Leung DY. Superantigens related to Kawasaki syndrome. Springer Semin Immunopathol 1996;17:385-96.

7. Son MB, Newburger JW. Kawasaki disease. Pediatr Rev 2013;34:151-62.

8. Tse SM, Silverman ED, McCrindle BW, Yeung RS. Early treatment with intravenous immunoglobulin in patients with Kawasaki disease. J Pediatr 2002;140:4505.

9. Wilson N, Heaton P, Calder L, et al. Kawasaki disease with severe cardiac sequelae: lessons from recent New Zealand experience. J Paediatr Child Health 2004;40:524-9.

10. Muise A, Tallett SE, Silverman ED. Are children with Kawasaki disease and prolonged fever at risk for macrophage activation syndrome? Pediatrics 2003;112:495.

11. Cummings $\mathrm{C}$, McCarthy $\mathrm{P}$, van Hoff $\mathrm{J}$, et al. Kawasaki disease associated with reactive hemophagocytic lymphohistiocytosis. Pediatr Infect Dis J 2008;27:1116-8.

12. Sano T, Kurotobi S, Matsuzaki K, et al. Predition of non-responsiveness to standard high-dose gamma-globulin therapy in patients with acute Kawasaki disease before starting initial treatment. Eur $\mathrm{J}$ Pediatr 2007;166:131-7.

13. Onouchi Y. Susceptibility genes for Kawasaki disease. Nihon Rinsho. 2014;72:1554-60.

14. Kobayashi T, Inoue $\mathrm{Y}$, Takeuchi $\mathrm{K}$, et al. Prediction of intravenous immunoglobulin unresponsiveness in patients with Kawasaki disease. Circulation 2006;113: 2606-12.

15. Newburger JW, Takahashi M, Gerber MA, et al. Diagnosis, treatment, and long-term management of Kawasaki disease: a statement for health professionals from the Committee on Rheumatic Fever, Endocarditis, and Kawasaki Disease, Council on Cardiovascular Disease in the Young, American Heart Association. Pediatrics 2004;114:1708-33.

16. Egami K, Muta H, Ishii M, et al. Prediction of resistance to intravenous immunoglobulin treatment in patients with Kawasaki disease. J Pediatr 2006;149:237-40.

17. Suresh N, Sankar J. Macrophage activation syndrome: a rare complication of incomplete Kawasaki disease. Ann Trop Paediatr 2010;30;61-4.

18. Taubert KA, Rowley AH, Shulman ST. Nationwide survey of Kawasaki disease and acute rheumatic fever. J Pediatr 1991;119:279-82.

19. Kato H, Sugimura T, Akagi T, et al. Longterm consequences of Kawasaki disease. A 10- to 21-year follow-up study of 594 patients. Circulation 1996;94:1379-85.

20. Dajani AS, Taubert KA, Gerber MA, et al. Diagnosis and therapy of Kawasaki disease in children. Circulation 1993;87:1776-80.

21. Suzuki A, Kamiya T, Tsuchiya K, et al. Tricuspid and mitral regurgitation detected by color flow Doppler in the acute phase of Kawasaki disease. Am J Cardiol 1988;61:386-90.

22. Hashino K, Ishii M, Iemura M, et al. Retreatment for immune globulin-resistant Kawasaki disease: a comparative study of additional immune globulin and steroid pulse therapy. Pediatr Int 2001;43:211-7.

23. Ogata S, Ogihara Y, Honda T, et al. Corticosteroid pulse combination therapy for refractory Kawasaki disease: a randomized trial. Pediatrics 2012;129:e17-23.

24. Rowley AH. The complexities of the diagnosis and management of Kawasaki Disease. Infect Dis Clin North Am 2015;29:525-37.

25. Patel RM, Shulman ST. Kawasaki disease: a comprehensive review of treatment options. J Clin Pharm Ther 2015;40:620-5.

26. Gromm AA, Passo M. Macrophage activation syndrome in systemic juvenile rheumatoid arthritis. J Pediatr 1996;129: 750-4.

27. Tristano AG. Macrophage activation syndrome: a frequent but underdiagnosed complication associated with rheumatic diseases. Med Sci Monit 2008;14:R27-36. 